JOURNAL OF

FUNCTION SPACES AND APPLICATIONS

Volume 9, Number 3 (2011), 217-244 (c) 2011, Scientific Horizon

http://www.jfsa.net

\title{
Generalization of I.Vekua's integral representations of holomorphic functions and their application to the Riemann-Hilbert-Poincaré problem
}

\author{
Vakhtang Kokilashvili and Vakhtang Paatashvili
}

(Communicated by Lars-Erik Persson)

2000 Mathematics Subject Classification. 30E20, 45E20, 47B38, 30E25.

Keywords and phrases. Holomorphic function, Cauchy type integral, variable exponent Lebesgue space, piecewise-smooth boundary, Riemann-HilbertPoincaré problem.

\footnotetext{
Abstract. I. Vekua's integral representations of holomorphic functions, whose $m$-th derivative $(m \geq 0)$ is Hölder-continuous in a closed domain bounded by the Lyapunov curve, are generalized for analytic functions whose $m$-th derivative is representable by a Cauchy type integral whose density is from variable exponent Lebesgue space $L^{p(\cdot)}(\Gamma ; \omega)$ with power weight. An integration curve is taken from a wide class of piecewise-smooth curves admitting cusp points for certain $p$ and $\omega$. This makes it possible to obtain analogues of I. Vekua's results to the Riemann-Hilbert-Poincaré problem under new general assumptions about the desired and the given elements of the problem. It is established that the solvability essentially depends on the geometry of a boundary, a weight function $\omega(t)$ and a function $p(t)$.
}

\section{Introduction}

In many boundary value problems of function theory the boundary conditions contain not only the sought function, but also its derivatives up to certain order. Therefore it is useful to have formulas giving an 
integral representation of this holomorphic function. One form of such representations, quite convenient for applications, was proposed by I. Vekua ([17], [18]). N. Muskhelishvili expounded them in his book, where they are called I. Vekua's integral representations (see [15, pp. 224-232]). This is I. Vekua's result.

Theorem (I. Vekua). Let $D^{+}$be a finite domain bounded by a simple closed Lyapunov curve $\Gamma$ and $\Phi(z)$ be a holomorphic function in $D^{+}$, whose derivative of order $m$ is continuous in $\overline{D^{+}}$and the boundary belong to the Hölder's class $H$. Then, assuming that the origin is in $D^{+}$, the function $\Phi$ is representable for $m=0$ as

$$
\Phi(z)=\int_{\Gamma} \frac{\varphi(t) d t}{1-\frac{z}{t}}+i d
$$

and for $m \geq 1$ as

$$
\Phi(z)=\int_{\Gamma} \varphi(t)\left(1-\frac{z}{t}\right)^{m-1} \ln \left(1-\frac{z}{t}\right) d s+\int_{\Gamma} \varphi(t) d s+i d,
$$

where $\varphi(t)$ is a real function from the class $H$, and $d$ is a real constant; $\varphi(t)$ and $d$ are defined uniquely with respect to $\Phi(z)$.

Subsequently, B. Khvedelidze [5] gave a generalization of this theorem to the case where a derivative of order $m$ of the function $\Phi(z)$ is representable in $D^{+}$by a Cauchy type integral with a density from the Lebesgue space $L^{p}(\Gamma ; \omega)$, where $p>1$ and

$$
\omega(t)=\prod_{k=1}^{n}\left|t-t_{k}\right|^{\alpha_{k}}, \quad t_{k} \in \Gamma, \quad-\frac{1}{p}<\alpha_{k}<\frac{1}{p^{\prime}}, \quad p^{\prime}=\frac{p}{p-1} .
$$

In that case, $\varphi$ belongs to $L^{p}(\Gamma ; \omega)$. In [1] K. Aptsiauri generalized this result to the case $\omega(t)=1$ for a wide subclass of piecewise-smooth curves.

I. Vekua used these representations for investigating quite a general boundary value problem, namely, the Riemann-Hilbert-Poincaré problem. An analogous investigation was carried out in [1] and [5].

In recent years, the attention of researchers has been attracted by Lebesgue spaces with a variable exponent $p(t)$ and the boundary value problems where the boundary values of the sought functions belong to these spaces (see, for instance, [3]-[4], [6]-[14]. Thus it is desirable to have representations (1)-(2) of holomorphic functions $\Phi$ with boundary values having this property and for as wide as possible classes of curves $\Gamma$, functions $p(t)$ and weights $\omega(t)$. 
In this paper, we consider the question whether integrals of form (1)(2) can be used to present holomorphic functions whose $m$-th derivative is representable by a Cauchy type integral with a density from $L^{p(\cdot)}(\Gamma ; \omega)$, where $\Gamma$ is a piecewise-smooth curve. Relations are found between the values of the function $p(t)$ at the angular points of the curve $\Gamma$, the values of these angles and the power exponents of weight functions, which provide the required representation.

Of the function $p(t)$ it is only required that it satisfies the Log-Hölder's condition which we frequently encounter in the literature. For the weight function $\omega(t)$ it is assumed that $\alpha_{k} \in\left(-\frac{1}{p\left(t_{k}\right)}, \frac{1}{p^{\prime}\left(t_{k}\right)}\right), p^{\prime}(t)=\frac{p(t)}{p(t)-1}$. The latter condition is necessary and sufficient for a singular operator with the Cauchy kernel to be continuous in the space $L^{p(\cdot)}(\Gamma ; \omega)$ (see [13], [10]) without which we can hardly do in our consideration. As a result, the range of domains in which representations (1)-(2) are valid has become much wider. This has been achieved thanks to the well studied Riemann-Hilbert problem in the considered classes. For this we had to extend the results obtained in [7], [8] for bounded domains to the case of unbounded domains. Also note that in [1], [5], [17]-[18] only the existence of $\varphi$ and $d$ such that (1), (2) holds is proved. Our approach enables us to indicate a more concrete construction of the function $\varphi$ by means of the prescribed function $\Phi(z)$.

The representations obtained are used in investigating the RiemannHilbert-Poincaré problem under more general assumptions than in [1] and [5].

The statements we make in connection with this problem are seemingly analogous to the results in [1], [5], [17], [18], but, in fact, the relations between the values essentially depend on the geometry of the boundary $\Gamma$, multipliers $\left|t-t_{k}\right|^{\alpha_{k}}$ from a weight function $\omega(t)$, discontinuity points of the principal coefficient from the boundary condition, as well as on the values of a function $p(t)$ at the angular points of $\Gamma$ and at points $t_{k}$.

The results of the present paper were announced in [9].

\section{Main definitions, the notation and auxiliary statements}

$\mathbf{1}^{0}$. Let $t=t(s), 0 \leq s \leq l$, be an equation of a simple rectifiable curve $\Gamma$ with respect to an arc abscissa. Let, further, $p(t)$ be a positive measurable function on $\Gamma$, and $\omega(t)$ be a measurable, a.e. finite and nonzero function. We denote by $L^{p(\cdot)}(\Gamma ; \omega)$ the set of measurable functions $f$ on $\Gamma$ for which 
$\|f\|_{L^{p(\cdot)(\Gamma ; \omega)}}<\infty$, where

$$
\|f\|_{L^{p(\cdot)}(\Gamma ; \omega)}=\inf \left\{\lambda>0: \int_{0}^{l}\left|\frac{f(t(s)) \omega(t(s))}{\lambda}\right|^{p(t(s))} d s \leq 1\right\} .
$$

It is assumed that $L^{p(\cdot)}(\Gamma ; 1)=L^{p(\cdot)}(\Gamma)$.

If

$$
\inf _{t \in \Gamma} p(t)=p_{-}>1, \quad \sup _{t \in \Gamma} p(t)=p_{+}<\infty,
$$

then $L^{p(\cdot)}(\Gamma ; \omega)$ is a Banach space with norm defined by (3).

$\mathbf{2}^{\mathbf{0}}$. Definition 1. We say that a measurable function $p$ on $\Gamma$ belongs to the class $\widetilde{\mathcal{P}}(\Gamma)$ if $p_{-}>1$ and, moreover, there exist positive constants $A$ and $\varepsilon$ such that

$$
\left|p\left(t_{1}\right)-p\left(t_{2}\right)\right|<\frac{A}{|\ln | t_{1}-t_{2}||^{1+\varepsilon}}
$$

for arbitrary $t_{1}$ and $t_{2}$ on $\Gamma$. The set of functions $p$ for which $p_{-}>1$ and equality (4) is fulfilled for $\varepsilon=0$ is denoted by $\mathcal{P}(\Gamma)$. It is obvious that $\widetilde{\mathcal{P}}(\Gamma) \subset \mathcal{P}(\Gamma)$.

$\mathbf{3}^{\mathbf{0}}$. We say that $\Gamma$ is a curve of the class $C_{D}^{1}\left(A_{1}, \ldots, A_{i} ; \nu_{1}, \ldots, \nu_{i}\right)$ if $\Gamma$ is a simple closed piecewise-smooth curve with angular points $A_{k}, k=\overline{1, i}$, at which the values of angles with respect to the domain $D$ whose boundary is $\Gamma$ are equal to $\pi \nu_{k}$. If, additionally, $\Gamma$ is a piecewise-Lyapunov curve, then we say that $\Gamma$ is a curve of the class $C_{D}^{1, L}\left(A_{1}, \ldots, A_{i} ; \nu_{1}, \ldots, \nu_{i}\right)$. If $D^{+}$and $D^{-}$are simply connected domains with the common boundary $\Gamma$ and $\Gamma$ is a curve of the class $C_{D^{+}}^{1}\left(A_{1}, \ldots, A_{i} ; \nu_{1}, \ldots, \nu_{i}\right)$, then $\Gamma$ is a curve of the class $C_{D^{-}}^{1}\left(A_{1}, \ldots, A_{i} ; \lambda_{1}, \ldots, \lambda_{i}\right)$, where $\nu_{k}+\lambda_{k}=2, k=\overline{1, i}$.

$4^{0}$. Definition 2. Let $D$ be a simply connected domain bounded by a simple rectifiable curve $\Gamma$, and the functions $p$ and $\omega$ be such that $L^{p(\cdot)}(\Gamma ; \omega) \subset L(\Gamma)$. We denote

$$
K_{D}^{p(\cdot)}(\Gamma ; \omega)=\left\{\Phi: \Phi(z)=\frac{1}{2 \pi} \int_{\Gamma} \frac{f(t) d t}{t-z}, \quad z \in D, \quad f \in L^{p(\cdot)}(\Gamma ; \omega)\right\} .
$$

We denote by $W^{p(\cdot)}(\Gamma)$ the set of weight functions $\omega$ for which the operator

$$
T_{\Gamma}: f \rightarrow T_{\Gamma} f, \quad\left(T_{\Gamma} f\right)(t)=\frac{\omega(t)}{\pi i} \int_{\Gamma} \frac{1}{\omega(\tau)} \frac{f(\tau)}{\tau-t} d \tau, \quad t \in \Gamma,
$$

is continuous in $L^{p(\cdot)}(\Gamma)$. 
If $\omega \in W^{p(\cdot)}(\Gamma)$, then every function $\Phi$ from the class $K_{D}^{p(\cdot)}(\Gamma ; \omega)$ has angular boundary values $\Phi^{+}(t)$ for almost all $t$ and $\Phi^{+} \in L^{p(\cdot)}(\Gamma ; \omega)$.

If $\Gamma$ is a Carleson curve and $p \in \mathcal{P}(\Gamma)$, then the function

$$
\omega(t)=\prod_{k=1}^{n}\left|t-t_{k}\right|^{\alpha_{k}}, \quad-\frac{1}{p\left(t_{k}\right)}<\alpha_{k}<\frac{1}{p^{\prime}\left(t_{k}\right)},
$$

belongs to $W^{p(\cdot)}(\Gamma)$ [13], [10]. For the case of constant $p$ we refer to [2].

Definition 3. If $m \geq 0$ is an integer number, then we denote by $K_{D, m}^{p(\cdot)}(\Gamma ; \omega)$ the set of functions $\Phi$ holomorphic in $D$ for which $\Phi^{(m)}(z) \in$ $K_{D}^{p(\cdot)}(\Gamma ; \omega)$. It is assumed that $\Phi^{(0)}(z)=\Phi(z)$ and thus $K_{D, 0}^{p(\cdot)}(\Gamma ; \omega)=$ $K_{D}^{p(\cdot)}(\Gamma ; \omega)$.

$5^{0}$. The problem of representation of holomorphic functions in terms of new assumptions reduces to the investigation of the Riemann-Hilbert problem in the class $K_{D^{-}}^{p(\cdot)}(\Gamma ; \omega)$. In [7] and [8], this problem is solved in the class $K_{D}^{p(\cdot)}(\Gamma ; \omega)$ when $D$ is a bounded domain with the boundary $\Gamma$. The case of an unbounded domain can be easily investigated by reducing it to the considered one.

In this subsection we recall the results from [7] and [8], and in Subsection $6^{0}$ extend them to the case of an unbounded domain.

Let: I. $\Gamma$ be a curve of the class $C_{D^{+}}^{1}\left(A_{1}, \ldots, A_{i} ; \nu_{1}, \ldots, \nu_{i}\right), 0<\nu_{k} \leq 2$, $k=\overline{1, i}, p \in \widetilde{\mathcal{P}}(\Gamma) ; a, b, c$ be real functions, $a$ and $b$ being piecewisecontinuous and $c \in L^{p(\cdot)}(\Gamma ; \omega)$, where $\omega$ is the weight function given by equality (5) or II. $\Gamma$ be a curve of the class $C_{D^{+}}^{1, L}\left(A_{1}, \ldots, A_{i} ; \nu_{1}, \ldots, \nu_{i}\right)$, $0<\nu_{k} \leq 2, k=\overline{1, i}, p \in \mathcal{P}(\Gamma) ; a$ and $b$ be piecewise-Hölder functions on $\Gamma$ and $c \in L^{p(\cdot)}(\Gamma ; \omega)$.

Let us consider the Riemann-Hilbert problem - Find a function $\Phi \in$ $K_{D^{+}}^{p(\cdot)}(\Gamma ; \omega)$ for which the equality

$$
\operatorname{Re}\left[(a(t)+i b(t)) \Phi^{+}(t)\right]=c(t)
$$

is valid a.e. on $\Gamma$.

We denote by $B_{k}, k=\overline{1, \lambda}$, the discontinuity points of the function $G(t)=-[a(t)-i b(t)][a(t)+i b(t)]^{-1}$ and assume that $\inf |G(t)|>0$ and $G\left(B_{k}^{-}\right) / G\left(B_{k}^{+}\right)=\exp \left(2 \pi i u_{k}\right)$.

Let $z=z(w)$ be the conformal mapping of the circle $U=\{w:|w|<1\}$ with boundary $\gamma=\{\tau:|\tau|=1\}$ on $D^{+}$and $w=w(z)$ be the inverse mapping. 
We assume

$T=\left\{\tau_{k}: \tau_{k}=w\left(t_{k}\right)\right\}, \quad A=\left\{a_{k}: a_{k}=w\left(A_{k}\right)\right\}, \quad B=\left\{b_{k}: b_{k}=w\left(B_{k}\right)\right\}$.

Let us numerate the points of the set $T \cup A \cup B$ as follows:

$$
\begin{aligned}
& w_{1}=\tau_{1}=a_{1}=b_{1}, \ldots, w_{\mu}=\tau_{\mu}=a_{\mu}=b_{\mu}, \\
& w_{\mu+1}=\tau_{\mu+1}=a_{\mu+1}, \ldots, w_{\mu+r}=\tau_{\mu+r}=a_{\mu+r}, \\
& w_{\mu+r+1}=\tau_{\mu+r+1}=b_{\mu+1}, \ldots, w_{\mu+r+q}=\tau_{\mu+r+q}=b_{\mu+q}, \\
& w_{\mu+r+q+1}=a_{\mu+r+1}=b_{\mu+q+1}, \ldots, w_{\mu+r+q+p}=a_{\mu+r+p}=b_{\mu+q+p}, \\
& w_{\mu+r+q+p+1}=\tau_{\mu+r+q+1}, \ldots, w_{\mu+r+q+p+M}=\tau_{\mu+r+q+M}, \\
& w_{\mu+r+q+p+M+1}=a_{\mu+r+p+1}, \ldots, w_{\mu+r+q+p+M+n}=a_{\mu+r+p+n}, \\
& w_{\mu+r+q+p+M+n+1}=b_{\mu+q+p+1}, \ldots, w_{\mu+r+q+p+M+n+s}=b_{\mu+p+q+s} .
\end{aligned}
$$

We set $\ell(\tau)=p(z(\tau))$ and write

(8)

$$
\delta_{k}=\left\{\begin{array}{lll}
\alpha_{k} \nu_{k}+\frac{\nu_{k}}{\ell\left(w_{k}\right)}+u_{k}, & k=\overline{1, \mu}, \\
\alpha_{k} \nu_{k}+\frac{\nu_{k}-1}{\ell\left(w_{k}\right)}, & k=\overline{\mu+1, \mu+r}, \\
\alpha_{k}+u_{k-r}, & k=\overline{\mu+r+1, \mu+r+q}, \\
\frac{\nu_{k-q}-1}{\ell\left(w_{k-q}\right)}+u_{k-r}, & k=\overline{\mu+r+q+1, \mu+r+q+p}, \\
\alpha_{k-p}, & k=\overline{\mu+r+q+p+1, \mu+r+q+p+M}, \\
\frac{\nu_{k-q-M}-1}{\ell\left(w_{k-q-M}\right)}, & k=\overline{\mu+r+q+p+M+1, \mu+r+q+p+M+n}, \\
u_{k-r-M-n}, & k=\overline{\mu+r+q+p+M+n+1, \mu+r+q+p+M+n+s} .
\end{array}\right.
$$

For a real number $x$ we set $x=[x]+\{x\}$, where $[x]$ is an integer number and $0 \leq\{x\}<1$.

Assume that

$$
\left\{\delta_{k}\right\} \neq \frac{1}{\ell^{\prime}\left(w_{k}\right)}, \quad k=\overline{1, j}, \quad j=\mu+r+q+p+M+n+s,
$$

and let

$$
\gamma_{k}= \begin{cases}{\left[\delta_{k}\right]} & \text { if }\left\{\delta_{k}\right\}<\frac{1}{\ell^{\prime}\left(w_{k}\right)}, \\ {\left[\delta_{k}\right]+1} & \text { if }\left\{\delta_{k}\right\}>\frac{1}{\ell^{\prime}\left(w_{k}\right)},\end{cases}
$$

and

$$
Q(w)=\prod_{k=1}^{j}\left(w-w_{k}\right)^{\gamma_{k}} .
$$


Furthermore, assuming that the point $z=0$ belongs to $D^{+}$, we write (see $[15$, p. 147])

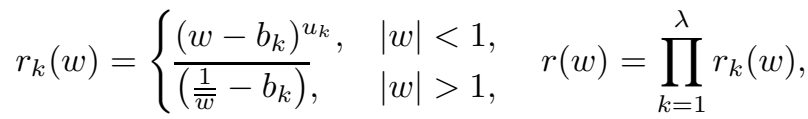

$$
\begin{aligned}
& R_{k}(\tau)=r_{k}^{+}(\tau)\left[r_{k}^{-}(\tau)\right]^{-1}=\exp \left(2 i \arg \left(\tau-b_{k}\right)\right), \\
& G_{1}(\tau)=G(z(\tau)) \prod_{k=1}^{\lambda} R_{k}(\tau), \quad \varkappa_{1}=\operatorname{ind} G_{1}(\tau), \quad \widetilde{G}_{1}(\tau)=G_{1}(\tau) \tau^{-\varkappa_{1}}, \\
& X_{1}(w)= \begin{cases}C \exp \left\{\frac{1}{2 \pi i} \int_{\gamma} \frac{\ln \widetilde{G}_{1}(\tau) d \tau}{\tau-w}\right\}, & |w|<1, \\
C w^{-\varkappa} \exp \left\{\frac{1}{2 \pi i} \int_{\gamma} \frac{\ln \widetilde{G}_{1}(\tau) d \tau}{\tau-w}\right\}, & |w|>1,\end{cases} \\
& X(w)=X_{1}(w) r(w), \quad C=\exp \left(-\frac{i \alpha}{2}\right), \quad \alpha=\frac{1}{2 \pi} \int_{0}^{2 \pi} q(\tau) d \tau, \\
& q(\tau)=\arg \left[-\tau^{-\varkappa_{1}} \arg G_{1}(\tau)\right] .
\end{aligned}
$$

We denote by $\varkappa_{0}$ the order of the rational function $Q(z)$ at infinity and assume that $\varkappa=\varkappa\left(\mu, G, \omega, D^{+}\right)=\varkappa_{0}+\varkappa_{1}$.

Then, by assumptions I (or II) and (9) we have

Proposition 1 ([7], [8]). If $\varkappa<-1$, then for problem (6) to be solvable in the class $K_{D^{+}}^{p(\cdot)}(\Gamma ; \omega)$ it is necessary and sufficient that the conditions

$$
\int_{\Gamma} \frac{c(t) Q(w(t)) w^{k}(t) w^{\prime}(t)}{X^{+}(w(t))(a(t)+i b(t))} d t=0, \quad k=\overline{0,|\varkappa|-2},
$$

be fulfilled, and their fulfilment implies that the problem has a unique solution

$$
\Phi(z)=\widetilde{\Omega}(w(z))=\frac{1}{2}\left(\Omega(w(z))+\Omega_{*}(w(z))\right),
$$

where

$\Omega(w)=X(w)[Q(w)]^{-1} \frac{1}{2 \pi i} \int_{\gamma} \frac{2 c(z(\tau)) Q(\tau)[a(z(\tau))+i b(z(\tau))]^{-1}}{X^{+}(\tau)(\tau-w)} d \tau, \quad|w| \neq 1$,

and

$$
\Omega_{*}(w)=\overline{\Omega\left(\frac{1}{\bar{w}}\right)}, \quad|w| \neq 1 .
$$

If $\varkappa \geq-1$, then problem (6) is certainly solvable and all its solutions are given by the equality

$$
\Phi(z)=\widetilde{\Omega}(w(z))+X(w(z))[Q(w(z))]^{-1} P_{\varkappa}(w(z)),
$$


where $P_{-1}(w) \equiv 0$, while for $\varkappa \geq 0$

$$
P_{\varkappa}(w)=h_{0}+h_{1} w+\cdots h_{\varkappa} w^{\varkappa}
$$

is an arbitrary polynomial whose coefficients $h_{k}$ satisfy the relation

$$
\bar{h}_{k}=A h_{\varkappa-k}, \quad k=\overline{0, \varkappa}, \quad A=(-1)^{\varkappa_{0}} \prod_{k=1}^{j} w_{k} .
$$

Remark 1. If we search for a solution $\Phi(z)$ of problem (6) with the condition $\Phi(0)=0$, then, as readily follows by the reasoning of [7], the above proposition remains valid when $\varkappa$ in it is replaced by $\varkappa-1$. Thus for $\varkappa<0$ the solvability conditions have the form

$$
\int_{\Gamma} \frac{c(t) Q(w(t)) w^{k}(t) w^{\prime}(t)}{X^{+}(w(t))[a(t)+i b(t)]} d t=0, \quad k=\overline{0,|\varkappa|-1}
$$

and their fulfillment implies that there exists a unique solution $\Phi(z)=$ $\widetilde{\Omega}(w(z))$.

When $\varkappa \geq 0$, the problem is solvable and all solutions are given by the equality

$$
\Phi(z)=\widetilde{\Omega}(w(z))+X(w(z))[Q(w(z))]^{-1} P_{\varkappa-1}(w(z)) .
$$

Remark 2. The investigation of problem (6) reduces to that of the Riemann problem

$$
\begin{gathered}
\Psi^{+}(\tau)=G(\tau) \Psi^{-}(\tau)+\frac{2 c(z(\tau))}{X^{+}(\tau)}, \quad G(\tau)=-\frac{\widetilde{a}(\tau)-\tilde{i} \widetilde{b}(\tau)}{\widetilde{a}(\tau)+\tilde{i b}(\tau)} \\
\widetilde{a}(\tau)=a(z(\tau)), \quad \widetilde{b}(\tau)=b(z(\tau)), \quad \tau \in \gamma
\end{gathered}
$$

in the class

$$
\begin{aligned}
& \widetilde{K}^{\widetilde{p}(\cdot)}(\gamma ; \omega) \\
= & \left\{\Psi: \Psi=\frac{1}{2 \pi i} \int_{\gamma} \frac{\psi(\tau) d \tau}{\tau-w}+\text { const }, w \notin \gamma, \quad \psi \in L^{\widetilde{p}(\cdot)}(\gamma ; \omega), \widetilde{p}(\cdot)=p(z(\tau))\right\}
\end{aligned}
$$

with the additional condition $\Psi(w)=\Psi_{*}(w)$, where $\Psi_{*}(w)=\overline{\Psi\left(\frac{1}{w}\right)}$, $|w| \neq 1$.

The function $Y(w)=X(w) Q(w)$ constructed in [7] (and in [8]) is a canonical function for $G(\tau)$. The order $\varkappa$ of the function $Y(w)$ at infinity 
is the index of the Riemann problem (12) (or, which is the same, the index of the singular operator $K \psi=\psi-\frac{1}{2}(G-1)\left(-\psi+S_{\gamma} \psi\right)$ in $L^{p(\cdot)}(\gamma ; \omega)$ (see [11])). Problem (6) is solvable if and only if problem (12), too, is solvable. Note that when $\varkappa>0$, the homogeneous problem (12) has a general solution depending on $\varkappa$ arbitrary complex constants, while the homogeneous problem $(6)$ has a general solution depending on $\varkappa$ arbitrary real constants (distinguished by relation (11)).

$6^{\mathbf{0}}$. Let $\Gamma$ be a curve of the class $C_{D^{-}}^{1}\left(A_{1}, \ldots, A_{i} ; \nu_{1}, \ldots, \nu_{i}\right), p \in \widetilde{\mathcal{P}}(\Gamma)$ and the Riemann-Hilbert problem be considered in the class $K_{D^{-}}^{p(\cdot)}(\Gamma ; \omega)$. This time the boundary condition is written in the form

$$
\operatorname{Re}\left[(a(t)+i b(t)) \Phi^{-}(t)\right]=c(t) .
$$

We assume that the point $z=0$ does not lie in $\bar{D}^{-}$. By transforming $\zeta=\frac{1}{z}$ the domain $D^{-}$becomes a finite domain which we denote by $D_{1}^{+}$. The function $\Phi(z)$ transforms to the function $F(\zeta)=\Phi\left(\frac{1}{\zeta}\right)$. Also $F(0)=0$. It is obvious that the boundary $\Gamma_{1}$ of the domain $D_{1}^{+}$is a curve of the class $C_{D_{1}^{+}}^{1}\left(\frac{1}{A_{1}}, \ldots, \frac{1}{A_{i}} ; \lambda_{1}, \ldots, \lambda_{i}\right), \lambda_{k}=2-\nu_{k}, k=\overline{1, i}$.

It is easy to verify that $p^{1}(\eta)=p\left(\frac{1}{\eta}\right)$ belongs to $\widetilde{\mathcal{P}}\left(\Gamma_{1}\right)$. The weight function $\omega(t)$ transforms to the function

$$
\omega^{1}(\eta)=\prod_{k=1}^{n}\left|\frac{1}{\eta}-\frac{1}{\eta_{k}}\right|^{\alpha_{k}}=\prod_{k=1}^{n} \frac{1}{\left|\eta \eta_{k}\right|^{\alpha_{k}}} \prod_{k=1}^{n}\left|\eta-\eta_{k}\right|^{\alpha_{k}} \sim \prod_{k=1}^{n}\left|\eta-\eta_{k}\right|^{\alpha_{k}}, \quad \eta_{k}=\frac{1}{t_{k}} \in \Gamma_{1},
$$

and therefore conditions (5) are fulfilled for $\omega^{1}(\eta)$.

The boundary condition (13) transforms to the condition

$$
\begin{gathered}
\operatorname{Re}\left[F^{+}(\eta)\left(a^{1}(\eta)+i b^{1}(\eta)\right)\right]=c_{1}(\eta) \\
a^{1}(\eta)=a\left(\frac{1}{\eta}\right), \quad b^{1}(\eta)=b\left(\frac{1}{\eta}\right), \quad c^{1}(\eta)=c\left(\frac{1}{\eta}\right) .
\end{gathered}
$$

It can be easily verified that $F \in K_{D_{1}^{+}}^{\left.p^{1} \cdot\right)}\left(\Gamma_{1} ; \omega^{1}\right)$. Thus we should solve problem (14) in the class $K_{D_{1}^{+}}^{p^{1} \cdot(}\left(\Gamma_{1} ; \omega^{1}\right)$ using the additional condition $F(0)=0$. If we require that $0<\lambda_{k} \leq 2$ (which is equivalent to the requirement $0 \leq \nu_{k}<2, k=\overline{1, i}$ ), then all the conditions required of $\Gamma_{1}, p^{1}, \omega^{1}$ in Subsection $5^{0}$ will be fulfilled. If $F(\zeta)$ is a solution of this problem, then $\Phi(z)=F\left(\frac{1}{z}\right)$ will be a solution of problem (13) of the class $K_{D^{-}}^{p(\cdot)}(\Gamma ; \omega)$. Thus we are to find a solution $F(\zeta)$ of problem (14) of the class $K_{D_{1}^{+}}^{p^{1}(\cdot)}\left(\Gamma_{1} ; \omega^{1}\right)$, where $\Gamma_{1}$ is a curve of the class $C_{D_{1}^{+}}^{1}\left(\frac{1}{A_{1}}, \ldots, \frac{1}{A_{i}} ; \lambda_{1}, \ldots, \lambda_{i}\right)$, $\lambda_{k}=2-\nu_{k}, k=\overline{1, i}$, with the condition $F(0)=0$. 
Let $\zeta=\zeta(w)$ be a conformal mapping of the circle $U$ onto $D_{1}^{+}$with the condition $\zeta(0)=0$, and $w=\psi(\zeta)$ be its inverse mapping. Besides, we denote

$$
G^{1}(\eta)=-\left[a^{1}(\eta)-i b^{1}(\eta)\right]\left[a^{1}(\eta)+i b^{1}(\eta)\right]^{-1}, \quad \ell(\tau)=p^{1}(\zeta(\tau))=p\left(\frac{1}{\zeta(\tau)}\right) .
$$

For the domain $D_{1}^{+}$we define the numbers $\omega_{k}^{1}, \delta_{k}^{1}$ and $\gamma_{k}^{1}$ by analogy with (7), (8) and (10). The numbers $\delta_{k}^{1}$ are obtained from formulas (8) in which $\nu_{k}$ is replaced by $\lambda_{k}$ (i.e., by $2-\nu_{k}$ ), while when defining the numbers $\gamma_{k}^{1}$, $\ell(\tau)$ is understood as $p\left(\frac{1}{\zeta(\tau)}\right)$. Define the function $X^{1}(w)$ and $Q^{1}(w)$ in accordance with the points $w_{k}^{1}$. Assume $\varkappa^{-}=\varkappa_{0}^{1}+\varkappa_{1}^{1}$.

Now, applying the result from Remark 1 of Subsection $5^{0}$, we obtain

Proposition 2. If $\Gamma$ is a curve of the class $C_{D^{-}}^{1}\left(A_{1}, \ldots, A_{i} ; \nu_{1}, \ldots, \nu_{i}\right)$, $0 \leq \nu_{k}<2, k=\overline{1, i}$, and problem (13) is considered in the class $K_{D^{-}}^{p(\cdot)}(\Gamma ; \omega)$, then, assuming that $\varkappa^{-}<0$, for the problem to be solvable it is necessary and sufficient that the conditions

$$
\int_{\Gamma_{1}} \frac{c^{1}(\eta) Q^{1}(\psi(\eta)) \psi^{k}(\eta) \psi^{\prime}(\eta)}{X^{1}(\psi(\eta))\left(a^{1}(\eta)+i b^{1}(\eta)\right)} d \eta=0, \quad k=\overline{0,\left|\varkappa^{-}\right|-1}
$$

is fulfilled, and when they are fulfilled, the problem has a unique solution

$$
\Phi(z)=\widetilde{\Omega}\left(\psi\left(\frac{1}{z}\right)\right)=\frac{1}{2}\left(\Omega\left(\psi\left(\frac{1}{z}\right)\right)+\Omega_{*}\left(\psi\left(\frac{1}{z}\right)\right)\right),
$$

where $\Omega_{*}(w)=\overline{\Omega\left(\frac{1}{w}\right)},|w| \neq 1$ and

$$
\Omega(w)=X_{1}^{1}(w)\left[Q^{1}(w)\right]^{-1} \frac{1}{2 \pi i} \int_{\gamma} \frac{2 c^{1}(\zeta(\tau)) Q^{1}(\tau)}{X^{1}(\tau)\left[a^{1}(\zeta(\tau))+i b^{1}(\zeta(\tau))\right]} d \tau, \quad|w| \neq 1 .
$$

When $\varkappa^{-} \geq 0$, the problem is unconditionally solvable and all solutions are given by the equality

$$
\Phi(z)=\widetilde{\Omega}\left(\psi\left(\frac{1}{z}\right)\right)+X^{1}\left(\psi\left(\frac{1}{z}\right)\right)\left[Q^{1}\left(\psi\left(\frac{1}{z}\right)\right)\right]^{-1} P_{\varkappa^{-}-1}\left(\psi\left(\frac{1}{z}\right)\right),
$$

where for the polynomial $P_{\varkappa^{-}-1}(w)=h_{0}+h_{1} w+\cdots+h_{\varkappa^{-}-1} w^{\varkappa^{-}-1}$ we have

$$
\bar{h}_{k}=A h_{\varkappa^{--k-1}}, \quad k=\overline{0, \varkappa^{-}-1}, \quad A=(-1)^{\varkappa_{0}} \prod_{k=1}^{j} w_{k}^{1} .
$$


If we reject the condition $\Phi(\infty)=0$, then, assuming that $\varkappa^{-}<-1$, for problem (13) to be solvable in the class $\widetilde{K}_{D^{-}}^{p(\cdot)}(\Gamma ; \omega)$, where $\widetilde{K}_{D^{-}}^{p(\cdot)}(\Gamma ; \omega)=$ $\left\{\Phi: \Phi=\Phi_{0}+\right.$ const, $\left.\Phi_{0} \in K_{D^{-}}^{p(\cdot)}(\Gamma ; \omega)\right\}$, it is necessary and sufficient that conditions (15) are fulfilled, where $k=\overline{0,|\varkappa|-2}$, and when they are fulfilled, there exists a unique solution given by equality (16).

When $\varkappa^{-} \geq-1$, the problem is unconditionally solvable and the solution is given by the equality

$$
\Phi(z)=\widetilde{\Omega}\left(\psi\left(\frac{1}{z}\right)\right)+X^{1}\left(\psi\left(\frac{1}{z}\right)\right)\left[Q^{1}\left(\psi\left(\frac{1}{z}\right)\right)\right]^{-1} P_{\varkappa^{-}}\left(\psi\left(\frac{1}{z}\right)\right),
$$

where, we recall, $\psi=\psi(\zeta)$ is a conformal mapping of $D_{1}^{+}$onto the unit circle $U ; P_{-1}(w)=0$, while when $\varkappa^{-} \geq 0$, conditions (11) are fulfilled for $P_{\varkappa^{-}}(w)$.

\section{I. Vekua's Integral Representation of Holomorphic Functions from the Class $K_{D^{+}, m}^{p(\cdot)}(\Gamma ; \omega)$}

$\mathbf{1}^{\mathbf{0}}$. In this subsection we will prove

\section{Theorem 1. Let}

(i) $\Gamma$ be a curve of the class $C_{D^{+}}^{1}\left(A_{1}, \ldots, A_{i} ; \nu_{1}, \ldots, \nu_{i}\right), 0 \leq \nu_{k}<2$, $k=\overline{1, i}$ and $p \in \widetilde{\mathcal{P}}(\Gamma)$ or $\Gamma \in C_{D^{+}}^{1, L}\left(A_{1}, \ldots, A_{i} ; \nu_{1}, \ldots, \nu_{i}\right), 0 \leq \nu_{k}<2$, $k=\overline{1, i}$ and $p \in \mathcal{P}(\Gamma)$;

(ii) $\omega$ be a power function of form (5);

(iii) the point $z=0$ lie in $D^{+} ; z=z(w)$ be a conformal mapping of the circle $U$ onto the domain $D^{-}=C D^{+} ; z(0)=\infty$ and $w=w(z)$ be its inverse mapping. Let $a_{k}=w\left(A_{k}\right), k=\overline{1, i}, \tau_{k}=w\left(t_{k}\right), k=\overline{1, n}$, and the points of the set $\left\{a_{1}, \ldots, a_{i}\right\} \cup\left\{\tau_{1}, \ldots, \tau_{n}\right\}$ be numbered so that

$$
\begin{aligned}
& w_{1}=a_{1}=\tau_{1}, \ldots, w_{\mu}=a_{\mu}=\tau_{\mu}, \\
& w_{\mu+1}=a_{\mu+1}, \ldots, w_{\mu+p}=a_{\mu+p}, \\
& w_{\mu+p+1}=\tau_{\mu+1}, \ldots, w_{\mu+p+M}=\tau_{\mu+M}
\end{aligned}
$$

and

$$
\delta_{k}= \begin{cases}\alpha_{k} \lambda_{k}+\frac{\nu_{k}-1}{\ell\left(w_{k}\right)}+\nu_{k}-1, & k=\overline{1, \mu}, \quad \lambda_{k}=2-\nu_{k}, \\ \frac{\nu_{k}-1}{\ell\left(w_{k}\right)}+\nu_{k}-1, & k=\overline{\mu+1, \mu+p}, \\ \alpha_{k-p}, & k=\overline{\mu+p+1, \mu+p+M},\end{cases}
$$

where $\ell(\tau)=p(z(\tau)),|\tau|=1$; 
(iv) $\Phi \in K_{D^{+}, m}^{p(\cdot)}(\Gamma ; \omega)$.

If

$\left\{\delta_{k}\right\} \neq \frac{1}{\ell^{\prime}\left(w_{k}\right)}, \quad k=\overline{1, j}, \quad j=n+i-\mu=\mu+p+M, \quad \ell^{\prime}(\tau)=\frac{\ell(\tau)}{\ell(\tau)-1}$,

then there exist a real function $\varphi \in L^{p(\cdot)}(\Gamma ; \omega)$ and a real constant $d$ such that

$$
\Phi(z)=\int_{\Gamma} \frac{\varphi(t) d s}{1-\frac{z}{t}}+i d
$$

for $m=0$,

$$
\Phi(z)=\int_{\Gamma} \varphi(t)\left(1-\frac{z}{t}\right)^{m-1} \ln \left(1-\frac{z}{t}\right) d s+\int_{\Gamma} \varphi(t) d s+i d
$$

for $m \geq 1$.

The function $\varphi$ and the constant $d$ are defined in a unique manner.

Before proceeding to the proof, we would like to note the particular cases of the theorem. Representations (20) and (21) are valid in the following cases:

I. a) $\Gamma$ is a smooth curve and $p \in \widetilde{\mathcal{P}}(\Gamma)$ or $\Gamma$ is a Lyapunov curve and $p \in \mathcal{P}(\Gamma)$;

b) $\omega$ is a weight function of form (5).

II. a) $\Gamma$ is a curve of the class $C^{1}\left(A_{1}, \ldots, A_{i} ; \nu_{1}, \ldots, \nu_{i}\right)$ and $p \in \widetilde{\mathcal{P}}(\Gamma)$ or $\Gamma$ is a curve of the class $C^{1, L}\left(A_{1}, \ldots, A_{i} ; \nu_{1}, \ldots, \nu_{i}\right)$ and $p \in \mathcal{P}(\Gamma)$ (in both cases $0<\nu_{k}<2, k=\overline{1, i}$ );

b) $\omega(t)=1$;

c) $\left\{\frac{\nu_{k}-1}{p\left(A_{k}\right)}+\nu_{k}-1\right\} \neq \frac{1}{p^{\prime}\left(A_{k}\right)}$.

Proof of Theorem 1. Since (20), (21) imply that $d=\operatorname{Im} \Phi(0)$, we can assume that $d=0$. Let us prove the validity of equalities (20) and (21). Having these representations for $d=0$, from the equality $\Phi(z)=[\Phi(z)-i \operatorname{Im} \Phi(0)]+i \operatorname{Im} \Phi(0)$ we will obtain the representations in the general case with $d=\operatorname{Im} \Phi(0)$.

Let $m=0$ and representation (20) hold. If $t=t(s)$ is the equation of the curve $\Gamma$ with respect to the arc abscissa, then $d s=\left(t^{\prime}\right)^{-1} d t=\overline{t^{\prime}} d t$ (see $[15$, p. 225]). Therefore (20) can be rewritten in the form

$$
\Phi(z)=\frac{1}{2 \pi i} \int_{\Gamma} \frac{2 \pi i t \overline{t^{\prime}} \varphi(t)}{t-z} d t .
$$


Since $\Phi \in K_{D^{+}}^{p(\cdot)}(\Gamma ; \omega)$ and $\omega \in W^{p(\cdot)}(\Gamma)$, we have $\Phi \in E^{1}\left(D^{+}\right)[11$, Theorem 3.3]. Therefore

$$
\Phi(z)=\frac{1}{2 \pi i} \int_{\Gamma} \frac{\Phi^{+}(t) d t}{t-z}, \quad \Phi^{+} \in L^{p(\cdot)}(\Gamma ; \omega) .
$$

Combined with (22), this implies that

$$
\frac{1}{2 \pi i} \int_{\Gamma} \frac{\Phi^{+}(t)-2 \pi i t \overline{t^{\prime}} \varphi(t)}{t-z} d t= \begin{cases}0, & z \in D^{+} \\ N(z), & z \in D^{-}\end{cases}
$$

Since $\Gamma$ is a piecewise-smooth curve, and $\Phi^{+}$and $\varphi$ belong to $L^{p(\cdot)}(\Gamma ; \omega)$, we have $N(z) \in E^{1}\left(D^{-}\right) \cap K_{D^{-}}^{p(\cdot)}(\Gamma ; \omega)$. Therefore from the latter equality it follows that

$$
\Phi^{+}(t)-2 \pi i t \overline{t^{\prime}} \varphi(t)=N^{-}(t)
$$

Let us divide both parts of equality (23) by $t \bar{t}$ and separate the real parts. We obtain

$$
\operatorname{Re} \frac{N^{-}(t)}{t \overline{t^{\prime}}}=\operatorname{Re} \frac{\Phi^{+}(t)}{t \overline{t^{\prime}}}, \quad t \in \Gamma
$$

We have come to the Riemann-Hilbert problem with regard to the function $N$ which we must solve in the class $K_{D^{-}}^{p(\cdot)}(\Gamma ; \omega)$.

For $m \geq 1$, from representation (21) we obtain

$$
\Phi^{(m)}(z)=(-1)^{m}(m-1) ! \int_{\Gamma} \frac{\varphi(t) \overline{t^{\prime}} d t}{t^{m-1}(t-z)}
$$

By the assumption of the theorem, $\Phi^{(m)} \in K_{D^{+}}^{p(\cdot)}(\Gamma ; \omega)$. This function, as mentioned above, belongs to $E^{1}\left(D^{+}\right)$. By this fact and $(25)$ we obtain

$$
\frac{1}{2 \pi i} \int_{\Gamma} \frac{\Phi^{(m)}(t)-2 \pi i(-1)^{m}(m-1) ! t^{1-m} \overline{t^{\prime}} \varphi(t)}{t-z} d t= \begin{cases}0, & z \in D^{+} \\ N(z), & z \in D^{-}\end{cases}
$$

where $N \in K_{D^{-}}^{p(\cdot)}(\Gamma ; \omega)$. From (25) we obtain

$$
\Phi^{(m)}(t)-2 \pi i(-1)^{m}(m-1) ! t^{1-m} \overline{t^{\prime}} \varphi(t)=N^{-}(t) .
$$

Therefore for $m \geq 1$ we have

$$
\operatorname{Re} \frac{N^{-}(t)}{t^{1-m} \overline{t^{\prime}}}=\operatorname{Re} \frac{\Phi^{(m)}(t)}{t^{1-m} \overline{t^{\prime}}} .
$$


For $m=0$ equality (27) coincides with (24).

If we solve problem $(27)$ in the class $K_{D^{-}}^{p(\cdot)}(\Gamma ; \omega)$, then, using equalities (23) and (26), we can define the function $\varphi$.

We will first show that representations (20) and (21) are unique. Both cases are treated in the same manner. Let, for instance,

$$
0=\int_{\Gamma} \frac{\varphi(t)}{1-\frac{z}{t}} d s+i d, \quad z \in D^{+} .
$$

For $z=0$ we have $\int_{\Gamma} \varphi(t) d s+i d=0$. Hence it follows that $d=0$. Let us prove that $\varphi(t)=0$. Expanding the integral $\int_{\Gamma} \frac{\varphi(t)}{1-\frac{z}{t}} d s$ into a series with respect to $z$ in the neighborhood of zero, we obtain

$$
\int_{\Gamma} \varphi(t) t^{-k} d s=0, \quad k=0,1, \ldots,
$$

i.e.

$$
\int_{\Gamma} \varphi(t) t^{k} \overline{t^{\prime}} d s=0, \quad k=0,-1,-2 \ldots
$$

By the Golubev-Privalov theorem, for the external domain [16, p. 202] we obtain $\varphi(t) \overline{t^{\prime}}=m^{-}(t)$, where $m(z) \in E^{1}\left(D^{-}\right)$. Further, absolutely in the same manner as in [1] we establish that $\lim _{z \rightarrow t, z \in D^{-}} \operatorname{Im} m_{0}(z)=0$, where $m_{0}(z)=\int_{z_{0}}^{z} m^{-}(z) d z, z_{0} \in D^{-}$, and therefore $m_{0}(z)=$ const, i.e., $m^{-}(z)=m_{0}^{\prime}(z)=0$. Hence we conclude that $\varphi(t)=0$.

Let us apply the results from Subsections $5^{0}$ and $6^{0}$ of the preceding section to problem (27).

In that case, $\Gamma_{1}$ is a curve of the class $C_{D_{1}^{+}}^{1}\left(\frac{1}{A_{1}}, \ldots, \frac{1}{A_{i}} ; 2-\nu_{1}, \ldots, 2-\nu_{i}\right)$, $a(t)+i b(t)=\frac{1}{t^{1-m} \overline{t^{\prime}}}, c(t)=\operatorname{Re} \frac{\Phi^{(m)}(t)}{t^{1-m} \overline{t^{\prime}}}$. Therefore

$$
G(t)=-\frac{a(t)-i b(t)}{a(t)+i b(t)}=-\frac{|a(t)+i b(t)|^{2}}{(a(t)+i b(t))^{2}}=-\frac{\left(t^{1-m} \overline{t^{\prime}}\right)^{2}}{\left|\left(t^{1-m} \overline{t^{\prime}}\right)^{2}\right|} .
$$

This function possesses discontinuity at the points $A_{k}, k=\overline{1, i}$. But then the function $G^{1}(\eta)=G\left(\frac{1}{\eta}\right)$ is discontinuous at the points $B_{k}^{1}=$ $\frac{1}{A_{k}} \in \Gamma_{1}$. It is not difficult to verify that $\varkappa_{1}^{1}=\operatorname{ind} G_{1}(\tau)=2 \mathrm{~m}$ and $G^{1}\left(B_{k}^{1}-\right) / G\left(B_{k}^{1}+\right)=\exp \left[-2 i\left(1-\nu_{k}\right) \pi\right]=\exp \left[2 i\left(\nu_{k}-1\right) \pi\right]$. Therefore $u_{k}=u_{k}^{1}=\nu_{k}-1$.

Furthermore, all discontinuity points of $G(t)$ coincide with the angular points of $\Gamma_{1}$. By assumption, on $\Gamma$ there exist $\mu$ angular points coinciding 
with some points $t_{k}$ from the weight $\omega$; accordingly, on $\Gamma_{1}$ there exist $\mu$ angular points coinciding with some points $\tau_{k}=\frac{1}{t_{k}}$ from the weight $\omega^{1}$. Furthermore, there are $p=i-\mu$ angular points not appearing among the singular points of the weight $\omega$. Finally, in the weight $\omega$ (in the weight $\left.\omega^{1}\right)$ there are $M$ multipliers of the form $\left(t-t_{k}\right)^{\alpha_{k}}\left(\left(\tau-\tau_{k}\right)^{\alpha_{k}}\right)$ in which the points $t_{k}\left(\tau_{k}\right)$ do not coincide with angular points. Let us assume that $\zeta=\zeta(w)$ is a conformal mapping of $U$ onto $D_{1}^{+}, \zeta(0)=0$ and $w=\psi(\zeta)$ is its inverse mapping. The function $z=\frac{1}{\zeta(w)}=z(w)$ gives the conformal mapping of $U$ onto $D^{-}$; let $w=w(z)\left(=\psi\left(\frac{1}{z}\right)\right)$ be the inverse mapping.

We have

$$
a_{k}=\zeta\left(\frac{1}{A_{k}}\right)=w\left(A_{k}\right), \quad \tau_{k}=\zeta\left(\frac{1}{t_{k}}\right)=w\left(t_{k}\right) .
$$

These points have been numbered according to (18). Then equalities (8) take form (19). Using these numbers, we define numbers $\gamma_{k}$ by means of equalities (10). Then $\varkappa_{0}^{1}=\sum_{k=1}^{j} \gamma_{k}, j=\nu+i-\mu$ and the index $\varkappa^{-}$of problem (13) is calculated by the equality $\varkappa^{-}=\varkappa(\Gamma ; m ; p ; \omega)=2 m+\sum_{k=1}^{j} \gamma_{k}$.

If $\varkappa^{-}<-1$, then the conditions of solvability of problem (27) in the class $K_{D^{-}}^{p(\cdot)}(\Gamma ; \omega)$ (or, which is the same, of problem (14) in the class $K_{D_{1}^{+}}^{p^{1}(\cdot)}\left(\Gamma_{1} ; \omega^{1}\right)$ with the condition $F(0)=0$ ) take the form

$$
\begin{gathered}
I_{k}=\int_{\Gamma_{1}} \frac{\operatorname{Re}\left[\Phi^{(m)}\left(\frac{1}{\eta}\right)\left(a^{1}(\eta)+i b^{1}(\eta)\right)\right]}{X^{1+}(\psi(\eta))\left(a^{1}(\eta)+i b^{1}(\eta)\right)} \\
k=\overline{0,\left|\varkappa^{-}\right|-1}
\end{gathered}
$$

Hence we obtain

$$
\begin{aligned}
I_{k} & =\frac{1}{2} \int_{\Gamma_{1}} \frac{\Phi^{(m)}\left(\frac{1}{\eta}\right)\left(a^{1}(\eta)+i b^{1}(\eta)\right)+\overline{\Phi^{(m)}\left(\frac{1}{\eta}\right)}\left(a^{1}(\eta)-i b^{1}(\eta)\right)}{X^{1+}(\psi(\eta))\left(a^{1}(\eta)+i b^{1}(\eta)\right)} Q^{1}(\psi(\eta)) \psi^{k}(\eta) \psi^{\prime}(\eta) d \eta \\
& =I_{k, 1}+I_{k, 2},
\end{aligned}
$$

where

$$
\begin{aligned}
I_{k, 1} & =\frac{1}{2} \int_{\Gamma_{1}} \frac{\Phi^{(m)}\left(\frac{1}{\eta}\right) Q^{1}(\psi(\eta))}{X^{1+}(\psi(\eta))} \psi^{k}(\eta) \psi^{\prime}(\eta) d \eta \\
I_{k, 2} & =\frac{1}{2} \int_{\Gamma_{1}} \frac{\overline{\Phi^{(m)}\left(\frac{1}{\eta}\right)} Q^{1}(\psi(\eta))}{X^{1+}(\psi(\eta))} \frac{\left(a^{1}(\eta)-i b^{1}(\eta)\right)}{a^{1}(\eta)+i b^{1}(\eta)} \psi^{k}(\eta) \psi^{\prime}(\eta) d \eta .
\end{aligned}
$$


The function $\Psi_{m}(\zeta)=\Phi^{(m)}\left(\frac{1}{\zeta}\right)\left[X^{1}(\psi(\zeta))\right]^{-1} Q^{1}(\psi(\zeta)) \psi^{k}(\zeta) \psi^{1}(\zeta)$ is holomorphic in $D_{1}^{+}$and belongs to $E^{\delta}\left(D_{1}^{+}\right)$for some $\delta>0$. Indeed, $\Phi^{(m)} \in E^{1}\left(D_{1}^{+}\right), \psi^{1} \in E^{1}\left(D_{1}^{+}\right),\left|\psi^{k}(\zeta)\right| \leq 1$. Furthermore $X^{1}(w)=$ $X_{1}^{1}(w) r(w)$ and $Q^{1}(w)$ is a rational function with zeros and poles on $\gamma$, $r(w)$ is a power function, while $X_{1}^{1} \in \bigcap_{\beta>0} E^{\beta}\left(D_{1}^{+}\right)$. Hence it follows that $Q^{1}(\psi(\zeta))\left[X^{1}(\psi(\zeta))\right]^{-1}$ and thereby $\Psi_{m}(\zeta)$, too, belongs to $E^{\delta}\left(D_{1}^{+}\right), \delta>0$. Moreover, since $\Gamma$ is a piecewise-smooth curve without zero angles (since $\left.0<2-\nu_{k} \leq 2\right)$, the construction of the functions $X^{1}$ and $Q^{1}$ implies that the function

$$
\Psi_{m}^{+}(\eta)=\Phi^{(m)}\left(\frac{1}{\eta}\right) Q^{1}(\psi(\eta))\left[X^{1+}(\psi(\eta))\right]^{-1} \psi^{k}(\eta) \psi^{\prime}(\eta)
$$

belongs to $L\left(\Gamma_{1}\right)$ (see [7, Theorem 2] and [8]). Thus, according to Smirnov's theorem, $\Psi \in E^{1}\left(D_{1}^{+}\right)$and therefore $I_{k, 1}=0$.

Let us write $I_{k, 2}$ in the form

$$
I_{k, 2}=\frac{1}{2} \int_{\gamma} \frac{\overline{\Phi^{(m)}\left(\frac{1}{\zeta(\tau)}\right)} Q^{1}(\tau)}{X^{1+}(\tau)} \frac{\left(a^{1}(\zeta(\tau))-i b^{1}(\zeta(\tau))\right)}{a^{1}(\zeta(\tau))+i b^{1}(\zeta(\tau))} \tau^{k} d \tau .
$$

But

$$
\frac{a^{1}(\zeta(\tau))-i b^{1}(\zeta(\tau))}{a^{1}(\zeta(\tau))+i b^{1}(\zeta(\tau))}=\frac{X^{1+}(\tau)}{X^{1-}(\tau)}
$$

and therefore

$$
I_{k, 2}=\frac{1}{2} \int_{\gamma} \frac{\overline{\Phi^{(m)}\left(\frac{1}{\zeta(\tau)}\right)} Q^{1}(\tau)}{X^{1-}(\tau)} Q^{1}(\tau) \tau^{k} d \tau .
$$

The function $\Phi^{(m)}\left(\frac{1}{\zeta(w)}\right)$ is holomorphic in the unit circle $U$ and therefore

$\overline{\Phi^{(m)}\left(\frac{1}{\zeta(\tau)}\right)}$ is a boundary value, on $\gamma$, of a holomorphic function $M^{-}$ in $U^{-}=\mathbb{C} \backslash \bar{U}$ (see $[15$, p. 143]). It is easy to verify that the functions $M^{-}(w)\left[X^{1}(w)\right]^{-1} Q^{1}(w) w^{k}, k=\overline{0,|\varkappa|-1}$, belong to $E^{1}\left(U^{-}\right)$. By virtue of this fact we have

$$
I_{k, 2}=\frac{1}{2} \int_{\gamma} \frac{M^{-}(\tau) Q^{1}(\tau)}{X^{1-}(\tau)} \tau^{k} d \tau=0, \quad k=\overline{0,|\varkappa|-1} .
$$

Thereby $I_{k}=0$ and thus conditions (28) sufficient for problem (27) to be solvable are fulfilled. The solution is given by equality (16). 
When $\varkappa^{-} \geq 0$, problem (13) is solvable in $K_{D^{-}}^{p(\cdot)}(\Gamma ; \omega)$ and the solution is given by the equality

$$
N(z)=\widetilde{\Omega}\left(\psi\left(\frac{1}{z}\right)\right)+X^{1}\left(\psi\left(\frac{1}{z}\right)\right)\left[Q^{1}\left(\psi\left(\frac{1}{z}\right)\right)\right]^{-1} P_{\varkappa^{--1}}\left(\psi\left(\frac{1}{z}\right)\right)
$$

$2^{0}$. Let us indicate how to define the function $\varphi$ when the solution of problem (27) is known. When the solution $N$ is unique, $\varphi$ is defined immediately from equalities (23) and (26). We will consider the case where $\varkappa^{-}>0$. By differentiation with respect to $z$, from (21) we find

$$
(-1)^{n} \frac{n !}{2 \pi i} \int_{\Gamma} \frac{2 \pi i(-1)^{m}(m-1) ! t^{1-m} \overline{t^{\prime}}}{(t-z)^{n+1}} \varphi(t) d t=\widetilde{\Omega}^{(m)}(z)+\sum_{k=0}^{\varkappa^{-}-1} h_{k}\left[w^{k}(z)\right]^{(n)} .
$$

Setting here $z=0, n=0,1, \ldots, \varkappa^{-}-1$, we obtain a system with respect to the unknowns $h_{0}, h_{1} \ldots, h_{\varkappa^{-}-1}, \varphi_{0}, \varphi_{1}, \ldots, \varphi_{\varkappa^{-}-1}$, where

$$
\varphi_{k}=\frac{(-1)^{k+1} k !}{2 \pi i} \int_{\Gamma} \frac{2 \pi i(-1)^{m}(m-1) ! t^{1-m} \overline{t^{\prime}}}{t^{k+1}} \varphi(t) d t .
$$

Thus we have $2 \varkappa^{-}$complex unknowns and $\varkappa^{-}$equations. To these equations we add $\varkappa^{-}$equations more: $\bar{h}_{k}=A h_{\varkappa^{-}-k-1}, k=\overline{0, \varkappa^{-}-1}$ (see (17)). As a result we obtain a linear system of $2 \varkappa^{-}$equations with respect to $2 \varkappa^{-}$unknowns. As has been proved above, there exists a unique desired solution $\varphi$. Therefore the obtained system is uniquely solvable. Substituting the numbers $h_{0}, \ldots, h_{\varkappa^{--1}}$ found from this solution into (29) we find $N(z)$ and then from (26) we define $\varphi$.

\section{The Riemann-Hilbert-Poincaré Problem in the Class

$$
K_{D^{+}, m}^{p(\cdot)}(\Gamma ; \omega)
$$

$\mathbf{1}^{\mathbf{0}}$. I. Vekua applied the representations (1), (2) to the investigation of the Riemann-Hilbert-Poincaré problem

$$
\operatorname{Re}\left[\sum_{k=0}^{m}\left(a_{k}\left(t_{0}\right) \Phi^{(k)}\left(t_{0}\right)+\int_{\Gamma} H_{k}\left(t_{0}, t\right) \Phi^{(k)}(t) d s\right)\right]=f\left(t_{0}\right),
$$

where $a_{k}, H_{k}, f$ are the given functions of Hölder's class, $\Gamma$ is a Lyapunov curve bounding the finite domain $D^{+}$, and the sought function $\Phi$ has a 
continuous derivative of order $m$ in $\overline{D^{+}}$and with boundary values from $H$ ([17], [18]). In [5] this problem is considered when $a_{k}(t)$ are continuous and $\Phi^{(m)}(z)$ is representable by a Cauchy type integral with a density from $L^{p}(\Gamma ; \omega)$, where $p>1$ and $\omega$ is a power function. For $\omega=1$, the problem is investigated for a wide subset of piecewise-smooth curves ([1]).

Here we assume that for $p, \Gamma$ and $\omega$ the conditions of Theorem 1 are fulfilled. We want to solve problem (30) in the class $K_{D^{+} . m}^{p(\cdot)}(\Gamma ; \omega)$, therefore it is assumed that $f \in L^{p(\cdot)}(\Gamma ; \omega)$. Since $\Phi^{(m)} \in K_{D^{+}}^{p(\cdot)}(\Gamma ; \omega) \subset E^{1}\left(D^{+}\right)$, the functions $\Phi^{(0)}(z)=\Phi(z), \Phi^{\prime}(z), \ldots, \Phi^{(m-1)}(z)$ are continuous in $\overline{D^{+}}$ and absolutely continuous on $\Gamma$ with respect to the arc abscissa (see, for instance, [16, p. 208]). Thus it is natural to assume that in condition (30) the coefficients $a_{k}(t), k=\overline{0, m-1}$, belong to $L^{p(\cdot)}(\Gamma ; \omega)$. As to the coefficient $a_{m}(t)$, we should assume that it is bounded. However this is not enough. Following [17], [18], [15], we reduce the problem to a singular integral equation in the class $L^{p(\cdot)}(\Gamma ; \omega)$, which is investigated in various conditions depending on the assumptions made for $p, \Gamma$ and $\omega$ ([4], [11]). It is assumed for simplicity that $a_{m}(t)$ is piecewise-continuous on $\Gamma$ and $\inf \left|a_{m}(t)\right|>0$.

So, let $\Gamma$ be a curve of the class $C_{D^{+}}^{1}\left(A_{1}, \ldots, A_{i} ; \nu_{1}, \ldots, \nu_{i}\right), 0 \leq \nu_{k}<2$, $\omega$ be the power function (5), the coefficients $a_{0}, a_{1}, \ldots, a_{m-1}$ belong to $L^{p(\cdot)}(\Gamma ; \omega), p \in \widetilde{\mathcal{P}}(\Gamma), a_{m} \in C\left(\widetilde{B}_{1}, \ldots, \widetilde{B}_{\lambda}\right)$ (i.e. $a_{m}$ is piecewise-continuous on $\Gamma$ with discontinuity points $\left.\widetilde{B}_{k}\right)$, and the operators

$$
\mathcal{H}_{k} \varphi=\int_{\Gamma} H_{k}\left(t_{0}, t\right) \varphi(t) d t, \quad t_{0} \in \Gamma,
$$

be compact in $L^{p(\cdot)}(\Gamma ; \omega)$.

It is required to find a function $\Phi \in K_{D^{+}, m}^{p(\cdot)}(\Gamma ; \omega)$ for which equality (30) holds a.e. on $\Gamma$. Note that the compactness of the operators $\mathcal{H}_{k}$ is provided, for instance, by the fulfillment of the conditions

$$
\left|H_{k}\left(t_{0}, t\right)\right|<\frac{A}{\left[s\left(t_{0}, t\right)\right]^{\lambda}}, \quad k=\overline{0, m},
$$

where $A, \lambda \in[0,1)$, are constants and $s\left(t_{0}, t\right)$ is the length of the smallest of two arcs connecting the points $t_{0}$ and $t$ on $\Gamma$ (see [12]).

Following $[15$, p. 233] this problem will sometimes be called Problem V.

$\mathbf{2}^{\mathbf{0}}$. Since under the above assumptions the conditions of Theorem 1 are fulfilled, the sought solution $\Phi$ is representable by equality (20) for $m=0$ and by equality (21) for $m \geq 1$.

Assuming first that $m \geq 1$, we calculate the derivatives of the function $\Phi$ given by equality (21) and substitute them into (30). Thus we obtain (see 
[15, pp. 234-235]) that the function $\varphi$ satisfies the condition

$$
N \varphi=A\left(t_{0}\right) \varphi\left(t_{0}\right)+\int_{\Gamma} N\left(t_{0}, t\right) \varphi(t) d s=f\left(t_{0}\right)-d \sigma\left(t_{0}\right)
$$

where

$$
\begin{aligned}
A\left(t_{0}\right)= & \operatorname{Re}\left[(-1)^{m}(m-1) ! \pi i t_{0}^{1-m} t_{0}^{\prime} a_{m}\left(t_{0}\right)\right] \\
\sigma\left(t_{0}\right)= & \operatorname{Re}\left[i a_{0}\left(t_{0}\right)+i \int_{\Gamma} h_{0}\left(t_{0}, t\right) d s\right], \\
N\left(t_{0}, t\right)= & \sum_{l=0}^{m} \operatorname{Re}\left[a_{l}\left(t_{0}\right) N_{l}\left(t_{0}, t_{1}\right) N_{l}\left(t_{1}, t\right) d s_{1}\right] \\
& +\operatorname{Re}\left[(-1)^{m}(m-1) ! \pi i h_{m}\left(t_{0}, t\right) t^{1-m} \overline{t^{\prime}}\right], \\
N_{0}\left(t_{0}, t\right)= & \left(1-\frac{t_{0}}{t}\right)^{m-1} \ln \left(1-\frac{t_{0}}{t}\right)+1, \quad N_{m}\left(t_{0}, t\right)=\frac{(-1)^{m}(m-1) !}{t^{m-1}\left(t-t_{0}\right)}, \\
N_{l}\left(t_{0}, t\right)= & (-1)^{l} \frac{(m-1) \cdots(m-l)}{t^{l}}\left(1-\frac{t_{0}}{t}\right)^{m-l-1} \\
& \times\left(\ln \left(1-\frac{t_{0}}{t}\right)+\frac{1}{m-1}+\cdots+\frac{1}{m-l}\right), \quad l=\overline{1, m-1} .
\end{aligned}
$$

It is evident that

$$
N \varphi=N^{0} \varphi+T \varphi
$$

where

$$
\begin{aligned}
N^{0} \varphi= & A\left(t_{0}\right) \varphi\left(t_{0}\right)+\frac{B\left(t_{0}\right)}{\pi i} \int_{\Gamma} \frac{\varphi(t) d t}{t-t_{0}} \\
B\left(t_{0}\right)= & \frac{1}{2}(-1)^{m}(m-1) ! \pi i\left[t_{0}^{1-m} \overline{t_{0}^{\prime}} a_{m}\left(t_{0}\right)+\overline{t_{0}^{1-m}} t_{0}^{\prime} \overline{a_{m}\left(t_{0}\right)}\right] \\
T \varphi= & \int_{\Gamma} \sum_{l=0}^{m-1} \operatorname{Re}\left[a_{l}\left(t_{0}\right) N_{l}\left(t_{0}, t\right)+\int_{\Gamma} H_{l}\left(t_{0}, t_{1}\right) N_{l}\left(t_{1}, t\right) d s_{1}\right] \varphi(t) d s \\
& +\int_{\Gamma} \operatorname{Re}\left[(-1)^{m}(m-1) ! \pi i H_{m}\left(t_{0}, t\right) t^{1-m} \overline{t^{\prime}}\right] \varphi(t) d s
\end{aligned}
$$

Moreover, (32) implies that the function $A\left(t_{0}\right)$ can be written in the form

$$
A\left(t_{0}\right)=\frac{1}{2}(-1)^{m}(m-1) ! \pi i\left[t_{0}^{1-m} \overline{t_{0}^{\prime}} a_{m}\left(t_{0}\right)-\overline{t_{0}^{1-m}} t_{0}^{\prime} \overline{a_{m}\left(t_{0}\right)}\right] .
$$


By virtue of our assumptions about $\Gamma$, the coefficients $a_{0}\left(t_{0}\right), \ldots, a_{m-1}\left(t_{0}\right)$ and operators $\mathcal{H}_{k}$ and using the above-mentioned result from [12] it is not difficult to establish that the operator $T$ is compact in $L^{p(\cdot)}(\Gamma ; \omega)$.

$3^{0}$. When $m=0$, the boundary condition (30) takes the form

$$
\operatorname{Re}\left[a_{0}\left(t_{0}\right) \Phi\left(t_{0}\right)+\int_{\Gamma} H_{0}\left(t_{0}, t\right) \Phi(t) d s\right]=f\left(t_{0}\right) .
$$

Applying representation (20) we come to equation (31), where $A(t), \sigma(t)$, $N\left(t_{0}, t\right)$ being calculated by the formulas given for them in preceding subsection, where we should take $m=0,(-1)^{m}(m-1) !=1$ and $N_{0}\left(t_{0}, t\right)=t\left(t-t_{0}\right)^{-1}$ (see [7, pp. 235-236]).

$4^{0}$. Let us calculate the index of the operator $N$ or, which is the same, of the operator $N^{0}$ (given by equality (33)). This is equivalent to the calculation of the index of the problem

$$
\Psi^{+}(t)=\frac{A(t)-B(t)}{A(t)+B(t)} \Psi^{-}(t), \quad \Psi(\infty)=\text { const }
$$

in the class $\widetilde{K}^{p(\cdot)}(\Gamma ; \omega)$ (see Remark 2 from Subsection $5^{0}$ of Section 2).

From (34) and (35) we obtain

$$
G(t)=\frac{A(t)-B(t)}{A(t)+B(t)}=-\frac{\overline{t^{1-m}} t^{\prime} a_{m}(t)}{t^{1-m} \overline{t^{\prime}} a_{m}(t)} .
$$

Denote by $B_{k}, k=\overline{1, \lambda}$, the discontinuity points of this function and let $b_{k}=w\left(B_{k}\right)$. Among the points $B_{k}$ there are all points $A_{k}, k=\overline{1, i}$.

Put

$$
-\frac{t^{m-1} t^{\prime}}{\overline{t^{m-1}} \overline{t^{\prime}}}=e^{-\vartheta(s)}, \quad \overline{\overline{a_{m}(t)}}=e^{i \theta(s)}
$$

and write equality (36) in the form

$$
\Psi^{+}(t)=\exp \{i(\vartheta(s)+\theta(s))\} \Psi^{-}(t) .
$$

The function $G(t)=G(t(s))=\exp \{i(\vartheta(s)+\theta(s))\}$ is piecewise-continuous. Let

$$
G\left(B_{k}-\right) / G\left(B_{k}+\right)=\exp \left(2 \pi i u_{k}\right) .
$$

For problem (36) the index $\varkappa$ (see Remark 2 from $5^{0}$ of Section 2), which depends on $\Gamma, p, \omega$ and the numbers $u_{k}$, is defined in the class $\widetilde{K}^{p(\cdot)}(\Gamma ; \omega)$. 
$5^{0}$. The operator

$$
\left(N^{\prime} g\right)(t)=A(t) g(t)+\int_{\Gamma} N\left(t_{0}, t\right) g\left(s_{0}\right) d s_{0}
$$

considered in the space $L^{\left.p^{\prime} \cdot \cdot\right)}\left(\Gamma ; \omega^{-1}\right)$ is the conjugate operator to $N$ (see (31)).

Let for a pair of operators $N$ and $N^{\prime}$ the Noether theorems hold (see, for instance, [4], [11]). We can assume that the conditions of Theorem 11 from [11] are fulfilled. From this theorem it follows particulary the following statement.

Let $\omega=\exp \left\{\frac{i}{2} S_{\Gamma} \varphi\right\}$, where $\varphi$ is a real piecewise-constant function,

$$
G_{\varphi}(t)=G(t) \exp (i \varphi(t)) \quad\left(G(t)=\frac{\overline{t^{1-m}} t^{\prime} \overline{a_{m}(t)}}{t^{1-m} \overline{t^{\prime}} a_{m}(t)}\right),
$$

and $B_{k, \varphi}, k=\overline{1, \lambda}$, are all discontinuity points of this function and $G\left(B_{k, \varphi}-\right) \times$ $\left[G\left(B_{k, \varphi}+\right)\right]^{-1}=\exp \left(2 \pi i \lambda_{k}\right), \lambda_{k}=\lambda_{k, 1}+i \lambda_{k, 2}, k=\overline{1, n_{\varphi}}$.

If $\left\{\lambda_{k, 1}\right\} \neq \frac{1}{p^{\prime}\left(B_{k, \varphi)}\right.}, k=\overline{1, n_{\varphi}}$, then the operator $N$ is Noetherian in $L^{p(\cdot)}(\Gamma ; \omega)$ and ind $N=\operatorname{ind} G_{\varphi}(t)=\varkappa$.

Theorem 2. Let the conditions of Subsection $1^{0}$ be fulfilled. Then for Problem $V$ to be solvable in the class $K_{D^{+}, m}^{p(\cdot)}(\Gamma ; \omega)$ it is necessary and sufficient that for some real $d$ the function $\widetilde{f}\left(t_{0}\right)=f\left(t_{0}\right)-d \sigma\left(t_{0}\right)$ should satisfy the conditions

$$
\int_{\Gamma} \widetilde{f}\left(t_{0}\right) g_{k}\left(s_{0}\right) d s_{0}=0, \quad k=\overline{1, n^{\prime}}
$$

where $g_{1}, \ldots, g_{n^{\prime}}$ are linearly independent solutions from the class $L^{p^{\prime}(\cdot)}\left(\Gamma ; \omega^{-1}\right)$ of the equation $N^{\prime} g=0$, where $N^{\prime}$ is the adjoint operator to the operator $N$.

In order that Problem $V$ has a solution, for any right-hand part of $f$ it is necessary and sufficient that $n^{\prime}=0$ or $n^{\prime}=1$ and in the latter case the solution $g$ of the equation $N^{\prime} g=0$ must satisfy the condition

$$
(g, \sigma)=\int_{\Gamma} g\left(t_{0}\right) \sigma\left(t_{0}\right) d s_{0} \neq 0 .
$$

In both cases the homogeneous problem has $\varkappa+1$ linearly independent solutions (where $\varkappa \geq-1$ ).

If these conditions are misobserved, then: if $\left(g_{k}, \sigma\right)=0$ for any $k=\overline{1, n^{\prime}}$, then the homogeneous problem has $\varkappa+n^{\prime}$ linearly independent solutions, 
and if among the numbers $\left(g_{k}, \sigma\right)$ there is at least one nonzero number, then it has $\varkappa+n^{\prime}+1$ solutions.

If $\sigma(t)=0$, then problem (30) is solvable for any right-hand part of $f\left(t_{0}\right)$ if and only if $n^{\prime}=0$; in that case the homogeneous problem has $\varkappa+1$ linearly independent solutions.

We omit the proof of this theorem because it is analogous to that of the respective I. Vekua's theorem ([18], see also [15, pp. 238-240]).

$\mathbf{6}^{0}$. If we assume

$$
\Psi(z)=\int_{\gamma} g(t) \Omega^{*}(t, z) d s, \quad z \in D^{-},
$$

where

$$
\Omega^{*}\left(t_{0}, z\right)=\sum_{k=0}^{m}\left\{a_{k}\left(t_{0}\right) N_{k}\left(t_{0}, z\right)+\int_{\Gamma} H_{k}\left(t_{0}, t\right) N_{k}(t, z) d s\right\},
$$

then the equation $N^{\prime} g=0$ can be written in the form

$$
\operatorname{Re} \Psi^{-}\left(t_{0}\right)=0
$$

It can be easily verified that $\Psi(z)$ belongs to $\widetilde{K}_{D^{-}}^{p^{\prime} \cdot(}\left(\Gamma ; \omega^{-1}\right)$. Thus the function $\Psi$ defined by equality (37) is a solution of the class $\widetilde{K}_{D^{-}}^{p^{\prime}(\cdot)}\left(\Gamma ; \omega^{-1}\right)$ of problem (38). In order to apply Proposition 1 given above to the case in which $p(t)$ is replaced by $p^{\prime}(t)$ and $\omega(t)$ by $[\omega(t)]^{-1}$, we have to calculate the index $\varkappa=\varkappa_{0}+\varkappa_{1}$ of problem (38). Since for the considered RiemannHilbert problem $a(t)+i b(t)=1$, we have $\varkappa_{1}=0$. As to $\varkappa_{0}$, it is equal to the order at the point $z=\infty$ of the rational function

$$
Q(w)=\prod_{k=1}^{j}\left(w-w_{k}\right)^{\gamma_{k}}
$$

Here the numbers $\gamma_{k}$ are defined from relations (10) in which the function $\ell(t)$ is replaced by the function $\ell^{\prime}(t)$ and the numbers $\alpha_{k}$ by the numbers $\left(-\alpha_{k}\right)$. The number $\varkappa_{0}$ is calculated by the equality

$\varkappa_{0}=\mathcal{N}\left\{A_{k}: p^{\prime}\left(A_{k}\right)<\nu_{k}\right\}+\mathcal{N}\left\{t_{k}=A_{k}: \frac{p^{\prime}\left(A_{k}\right)}{1-\alpha_{k} p^{\prime}\left(A_{k}\right)}<\nu_{k}<\frac{2 p^{\prime}\left(A_{k}\right)}{1-\alpha_{k} p^{\prime}\left(A_{k}\right)}\right\}$,

where $\mathcal{N}(E)$ stands for the number of elements of the set $E$ [7, Subsect. 7].

Problem (38) has $\varkappa_{0}+1$ linearly independent solutions. We denote them by $\Psi_{0}(z), \Psi_{1}(z), \ldots, \Psi_{\varkappa_{0}}(z)$. 
Let

$$
\widetilde{\Omega}\left(t_{0}, z\right)=\Omega^{*}\left(t_{0}, z\right)-\Psi_{0}(z)-\cdots-\Psi_{\varkappa_{0}}(z)
$$

and

$$
\Omega\left(t_{0}, z\right)=\widetilde{\Omega}\left(t_{0}, z\right)-i \operatorname{Im} \widetilde{\Omega}\left(t_{0}, \infty\right) .
$$

Then from the definitions of the functions $\Omega^{*}, \Psi_{0}, \ldots, \Psi_{\varkappa_{0}}$ it follows that for an arbitrary solution $g$ of the equation $N^{\prime} g=0$ there holds the equality

$$
\int_{\Gamma} g(t) \Omega(t, z) d s=0 \quad \forall z \in D^{-} .
$$

By analogy with $\left[15\right.$, p. 242] we call the function $\Omega\left(t_{0}, z\right)$ a kernel.

Now, following I. Vekua ([18], see also [15, pp. 242-243]), condition (41) can be replaced by an equivalent condition

$$
\int_{\Gamma} g(t) \omega_{j}(t) d s=0
$$

where $\omega_{j}(t)(j=0,1, \ldots)$ can be understood as any of the following systems of functions:

$$
\omega_{j}(t)=\Omega\left(t, z_{j}\right), \quad z_{j} \in D^{-},
$$

where $z_{0}, z_{1}, \ldots$ is an arbitrary sequence having a limit point in $D^{-}$;

$$
\omega_{j}(t)=\left[\frac{d^{j} \Omega(t, z)}{d z^{j}}\right]_{z=z_{0}},
$$

where $z_{0}$ is an arbitrary fixed point in $D^{-}$;

$$
\omega_{j}(t)=X_{j}(t), \quad j=0,1,2, \ldots,
$$

where $X_{0}(t)=\operatorname{Re}\left[a_{0}\left(t_{0}\right)+\int_{\Gamma} H_{0}\left(t_{0}, t\right) d s\right], X_{k}\left(t_{0}\right)=L \psi, \psi=t^{k}$, $k=1,2, \ldots$,

$$
L \psi=\sum_{j=0}^{m}\left\{a_{j}\left(t_{0}\right) \psi^{(j)}\left(t_{0}\right)+\int_{\Gamma} H_{j}\left(t_{0}, t\right) \psi^{(j)}(t) d s\right\} .
$$

If $k^{\prime}$ is a maximal number of linearly independent real functions $g_{j}(t)$ orthogonal to the kernel $\Omega(t, z)$ or to all elements of the sequence $\left\{\omega_{j}(t)\right\}$, then $k^{\prime}$ is called the defect of the kernel or of the sequence $\left\{\omega_{j}(t)\right\}$. In 
the considered case, $k^{\prime}$ coincides with $n^{\prime}$ which is the number of linearly independent solutions in $L^{p^{\prime}(\cdot)}\left(\Gamma ; \omega^{-1}\right)$ of the equation $N^{\prime} g=0$. Hence the obtained results can be formulated as follows:

For Problem $V$ to have a solution for any right-hand part of $f(t)$ it is necessary and sufficient that the defect of the sequence $\left\{\omega_{j}(t)\right\}$ (or of the kernel $\Omega(t, z)$ ) be equal to 0 or 1 . Note that in the latter case the function $g(t)$, which is orthogonal to all elements of the sequence $\left\{\omega_{j}(t)\right\}$, must not be orthogonal to the function $\sigma(t)$.

\section{Some Particular Cases}

$\mathbf{1}^{\mathbf{0}}$. Poincaré Problem. We will consider this problem formulated as follows. Find, in the domain $D^{+}$, a harmonic function $u$ from the set

$$
e_{D^{+}, 1}^{p(\cdot)}(\Gamma ; \omega)=\left\{u: u=\operatorname{Re} \Phi, \quad \Phi \in K_{D^{+}, 1}^{p(\cdot)}(\Gamma ; \omega)\right\},
$$

for which a.e. on $\Gamma$ we have

$$
\alpha(s) \frac{\partial u}{\partial n}+\beta(s) \frac{\partial u}{\partial s}+\gamma(s) u=f(s) .
$$

Here $\alpha(s), \beta(s), \gamma(s), f(s)$ are the real functions given on $\Gamma, s$ is an arc abscissa, $\frac{\partial u}{\partial n}$ is normal derivative. It is assumed that $p \in \widetilde{\mathcal{P}}(\Gamma)$, $\Gamma \in C^{1}\left(A_{1}, \ldots, A_{i} ; \nu_{1}, \ldots, \nu_{i}\right), 0<\nu_{k}<2, k=\overline{1, i} ; \alpha$ and $\beta$ belong to Hölder's class, while $\gamma$ and $f$ belong to $L^{p(\cdot)}(\Gamma ; \omega)$.

Let

$a(t)=-\alpha(s) \sin \vartheta(s)+\beta(s) \cos \vartheta(s), \quad b(t)=\alpha(s) \cos \vartheta(s)+\beta(s) \sin \vartheta(s)$,

where $\vartheta(s)$ is the angle formed between the tangent to $\Gamma$ at the point $t(s)$ and the axis of abscissa. Then condition (42) takes the form

$$
a(t) \frac{\partial u}{\partial x}+b(t) \frac{\partial u}{\partial y}+\gamma(s(t)) u=f(t), \quad t \in \Gamma,
$$

which can be rewritten as

$$
\operatorname{Re}\left[\left(a\left(t_{0}\right)+i b\left(t_{0}\right)\right) \Phi^{\prime}\left(t_{0}\right)+\gamma\left(t_{0}\right) \Phi\left(t_{0}\right)\right]=f\left(t_{0}\right), \quad t_{0} \in \Gamma .
$$


Using representation (21), for $m=1$ we obtain the equality

$$
\begin{aligned}
N \varphi= & \operatorname{Re}\left[-\pi i \overline{t_{0}^{\prime}}\left(a\left(t_{0}\right)+i b\left(t_{0}\right)\right)\right] \varphi\left(t_{0}\right) \\
& +\int_{\Gamma} \operatorname{Re}\left[\gamma\left(t_{0}\right) \ln e\left(1-\frac{t_{0}}{t}\right)-\frac{a\left(t_{0}\right)+i b\left(t_{0}\right)}{t-t_{0}}\right] \varphi(t) d s=f\left(t_{0}\right) .
\end{aligned}
$$

Let us assume that $\left(a^{2}+b^{2}\right)>0$ (or, which is the same, $\alpha^{2}+\beta^{2}>0$ ). This time we have

$$
\begin{aligned}
G(t) & =-\frac{t^{\prime}}{\overline{t^{\prime}}} \frac{\alpha(-\sin \vartheta-i \cos \vartheta)+\beta(\cos \vartheta-i \sin \vartheta)}{\alpha(-\sin \vartheta+i \cos \vartheta)+\beta(\cos \vartheta+i \sin \vartheta)} \\
& =-\frac{t^{\prime}}{\overline{t^{\prime}}} \frac{-\alpha i \overline{t^{\prime}}+\beta \overline{t^{\prime}}}{\alpha i t^{\prime}+\beta t^{\prime}}=-\frac{t^{\prime}}{\overline{t^{\prime}}} \frac{\overline{t^{\prime}}(\beta-\alpha i)}{t^{\prime}(\beta+\alpha i)}=\frac{\alpha+\beta i}{\alpha-\beta i} .
\end{aligned}
$$

Assume that

$$
n=\frac{1}{2 \pi}[\arg (\alpha(t)+i \beta(t))]_{\Gamma},
$$

where $[f]_{\Gamma}$ denotes an increment of the function $f(t)$ when the point $t$ performs one-time movement along the curve $\Gamma$. In that case the index of the operator $N$ (see (31)) is calculated by the equality $\varkappa=\varkappa_{0}+\varkappa_{1}$, where $\varkappa_{1}=2 n$ and

$$
\begin{aligned}
\varkappa_{0}= & \mathcal{N}\left\{A_{k}: A_{k} \notin \bigcup\left\{t_{j}\right\}, \nu_{k}>p\left(A_{k}\right)\right\} \\
& +\mathcal{N}\left\{t_{k}=A_{k}: \frac{p\left(A_{k}\right)}{1+\alpha_{k} p\left(A_{k}\right)}<\nu_{k}<\frac{2 p\left(A_{k}\right)}{1+\alpha_{k} p\left(A_{k}\right)}\right\}
\end{aligned}
$$

(recall that $A_{k}$ are the angular points of $\Gamma$ and $\alpha_{k}$ are power exponents from weight (5)). The kernel $\Omega\left(t_{0}, z\right)$ is given by equalities (39)-(40), where

$$
\Omega^{*}\left(t_{j}, z\right)=\frac{a\left(t_{0}\right)+i b\left(t_{0}\right)}{t_{0}-z}+\gamma\left(t_{0}\right) \ln e\left(1-\frac{t_{0}}{z}\right) .
$$

Besides, in our case $\sigma\left(t_{0}\right)=0$ and therefore by virtue of the above results we come to an analogue of I. Vekua's theorem ([18], see also [15, p. 245]).

Theorem 3. For the Poincaré problem to have a solution in the class $e_{D^{+}, 1}^{p(\cdot)}(\Gamma ; \omega)$ for any right-hand part of $f(t)$ it is necessary and sufficient that the equation

$$
\begin{aligned}
N^{\prime} g= & \operatorname{Re}\left\{-\pi i \overline{t_{0}^{\prime}}\left[a\left(t_{0}\right)+i b\left(t_{0}\right)\right]\right\} g\left(t_{0}\right) \\
& +\int_{\Gamma} \operatorname{Re}\left\{\gamma\left(t_{0}\right) \ln e\left(1-\frac{t_{0}}{t}\right)+\frac{a(t)+i b(t)}{t-t_{0}}\right\} g(t) d s=0
\end{aligned}
$$


would not have nonzero solutions in the class $L^{p^{\prime}(\cdot)}\left(\Gamma ; \omega^{-1}\right)$.

When this condition is fulfilled, the problem has $\varkappa+1$ linearly independent solutions, where $\varkappa=2 n+\varkappa_{0}$ ( $n$ is calculated by equality (43) and $\varkappa_{0}$ by equality (44)).

$\mathbf{2}^{\mathbf{0}}$. Neumann Problem in the Class $e_{D^{+}, 1}^{p(\cdot)}(\Gamma ; \omega)$. The Neumann problem

$$
\frac{\partial u}{\partial n}=f
$$

for the Laplace equation is a particular case of the Poincaré problem. It reduces to the problem

$$
\operatorname{Re}\left[i t_{0}^{\prime} \Phi^{\prime}\left(t_{0}\right)\right]=f\left(t_{0}\right)
$$

considered in the class $e_{D^{+}, 1}^{p(\cdot)}(\Gamma ; \omega)$.

The boundary value problem (38) reduces to the problem

$$
\Psi^{+}(t)=\Psi^{-}(t), \quad \Psi(\infty)=\text { const },
$$

whose conjugate problem is

$$
F^{+}(t)=F^{-}(t), \quad F \in \widetilde{K}^{p^{\prime}(\cdot)}\left(\Gamma ; \omega^{-1}\right) .
$$

Assuming that $p^{\prime}\left(A_{k}\right) \neq \nu_{k}$ holds for all $k$, the latter problem has $\varkappa\left(p^{\prime} ; \omega^{-1}\right)$ solutions, where

$$
\begin{aligned}
\varkappa\left(p^{\prime} ; \omega^{-1}\right)= & \mathcal{N}\left\{A_{k}: A_{k} \notin \bigcup\left\{t_{j}\right\}, p^{\prime}\left(A_{k}\right)<\nu_{k}\right\} \\
& +\mathcal{N}\left\{t_{k}=A_{k}: \frac{p^{\prime}\left(A_{k}\right)}{1-\alpha_{k} p^{\prime}\left(A_{k}\right)}<\nu_{k}<\frac{2 p^{\prime}\left(A_{k}\right)}{1-\alpha_{k} p^{\prime}\left(A_{k}\right)}\right\}
\end{aligned}
$$

(see [7]). Thus $\varkappa\left(p^{\prime} ; \omega^{-1}\right)+1$ conditions must be fulfilled in order that problem (45) be solvable.

When $\omega=1$, this number of conditions is equal to $\varkappa\left(p^{\prime} ; 1\right)$, where

$$
\varkappa\left(p^{\prime} ; 1\right)=\mathcal{N}\left\{A_{k}: p^{\prime}\left(A_{k}\right)<\nu_{k}\right\} .
$$

In that case for the Neumann problem we have $\varkappa\left(p^{\prime} ; 1\right)+1$ solvability conditions. Assuming that (9) holds and the solvability conditions are fulfilled, a general solution of problem $(45)$ contains $\varkappa(p ; 1)$ real constants, where

$$
\varkappa(p ; 1)=\mathcal{N}\left\{A_{k}: p\left(A_{k}\right)<\nu_{k}\right\} .
$$


Acknowledgement. This work was supported by grant no. GNSF/ST07/3169 of the Georgian National Science Foundation.

\section{References}

[1] K. Aptsiauri, On the generalized Riemann-Hilbert-Poincaré problem in domains with piecewise smooth boundaries, Proc. A. Razmadze Math. Inst., 114 (1997), 3-26.

[2] A. Böttcher and Yu. I. Karlovich, Carleson Curves, Muckenhoupt Weights, and Toeplitz Operators, 154 of Progress in Mathematics, Birkhäuser Verlag, Basel, 1997.

[3] L. Diening and M. Ri̊zička, Calderon-Zygmund operators on generalized Lebesgue spaces $L^{p(\cdot)}$ and problems related to fluid dynamics, J. Reine Angew. Math., 563 (2003), 197-220.

[4] A. Yu. Karlovich, Fredholmness of singular integral operators with piecewise continuous coefficients on weighted Banach function spaces, J. Integral Equations Appl., 15 (2003), 263-320.

[5] B. V. Khvedelidze, Linear discontinuous boundary problems in the theory of functions, singular integral equations and some of their applications, (Russian) Trudy Tbiliss. Mat. Inst. Razmadze, 23 (1956), 3-158.

[6] V. Kokilashvili and V. Paatashvili, The Dirichlet problem for harmonic functions in the Smirnov class with variable exponent, Georgian Math. J., 14 (2007), 289-299.

[7] V. Kokilashvili and V. Paatashvili, The Riemann-Hilbert problem in weighted classes of Cauchy type integrals with density from $L^{p(\cdot)}(\Gamma)$, Complex Anal. Oper. Theory, 2 (2008), 569-591.

[8] V. Kokilashvili and V. Paatashvili, The Riemann-Hilbert problem in a domain with piecewise smooth boundaries in weight classes of Cauchy type integrals with a density from variable exponent Lebesgue spaces, Georgian Math. J. 16 (2009), 737-755.

[9] V. Kokilashvili and V. Paatashvili, On the Riemann-Hilbert-Poincaré problem and I. Vekua's representation of holomorphic functions, Bull. Georgian Nat. Acad. Sci., 3 (2009), 25-29.

[10] V. Kokilashvili, V. Paatashvili and S. Samko, Boundedness in Lebesgue spaces with variable exponent of the Cauchy singular operator on Carleson curves, in Modern operator theory and applications, 167-186, vol. 170 of Oper. Theory Adv. Appl., Birkhäuser, Basel, 2007. 
[11] V. Kokilashvili, V. Paatashvili and S. Samko, "Boundary value problems for analytic functions in the class of Cauchy-type integrals with density in $L^{p(\cdot)}(\Gamma)$," Bound. Value Probl., vol. 2005, no. 1, pp. 4371, 2005.

[12] V. Kokilashvili and S. Samko, Maximal and fractional operators in weighted $L^{p(x)}$ spaces, Rev. Mat. Iberoamericana, 20 (2004), 493-515.

[13] V. Kokilashvili and S. Samko, Weighted boundedness in Lebesgue spaces with variable exponents of classical operators on Carleson curves, Proc.

A. Razmadze Math. Inst., 138 (2005), 106-110.

[14] O. Kovačik and J. Rákosník, On spaces $L^{p(x)}$ and $W^{k, p(x)}$, Czechoslovak Math. J., 41 (116) (1991), 592-618.

[15] N. I. Muskhelishvili, Singular Integral Equations, 3rd ed. (Russian) Izdat. "Nauka", Moscow, 1968 (English translation from 1st Russian edition: P. Noordhoff N. V., Groningen, 1953).

[16] I. I. Privalov, Boundary Properties of Analytic Functions, 2nd ed. (Russian) Gosudarstv. Izdat. Tehn.-Teor. Lit., Moscow-Leningrad, 1950 .

[17] I. Vekua, On a new integral representation of analytic functions and its applications, (Russian) Bull. Acad. Sci. Georgian SSR, 2 (1941), 477-484.

[18] I. Vekua, On a linear boundary value problem of Riemann, (Russian) Trudy Tbiliss. Mat. Inst. Razmadze, XI (1942), 109-139.

A. Razmadze Mathematical Institute

1, M. Aleksidze St., Tbilisi 0193

Georgia

(E-mail : kokil@rmi.acnet.ge)

(E-mail : paatashvilitam@gmail.com)

(Received : September 2009) 


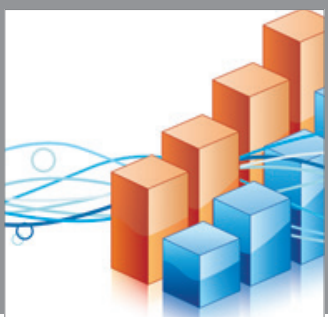

Advances in

Operations Research

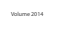

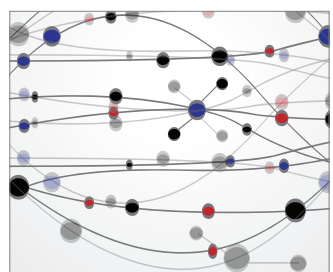

\section{The Scientific} World Journal
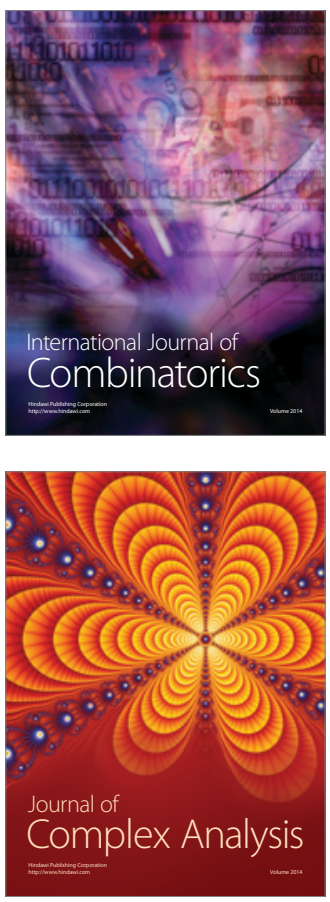

International Journal of

Mathematics and

Mathematical

Sciences
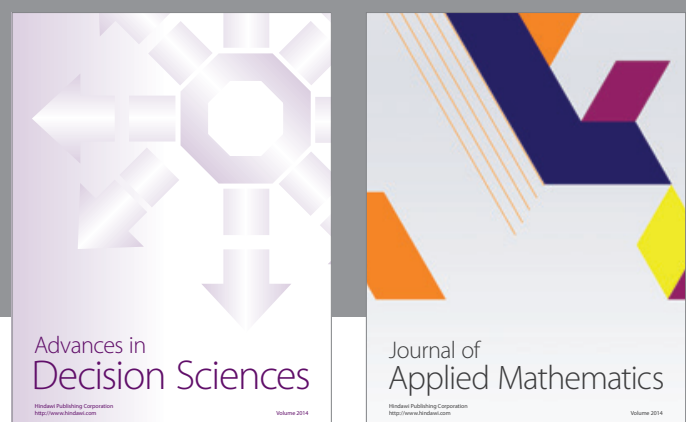

Journal of

Applied Mathematics
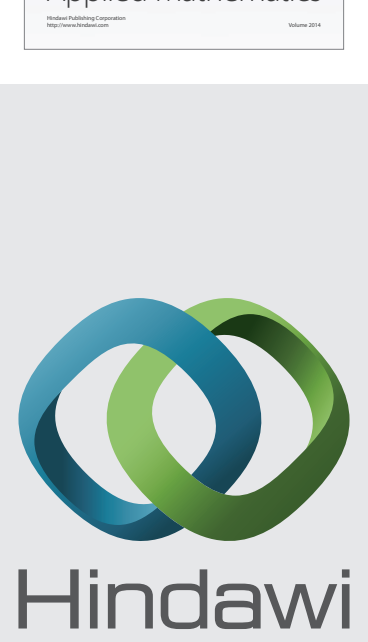

Submit your manuscripts at http://www.hindawi.com
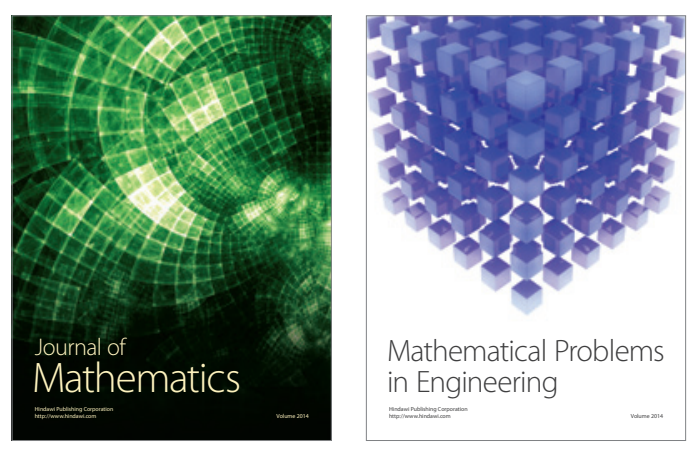

Mathematical Problems in Engineering
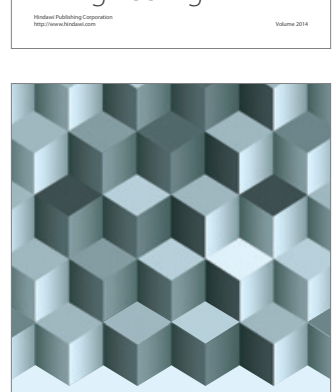

Journal of

Function Spaces
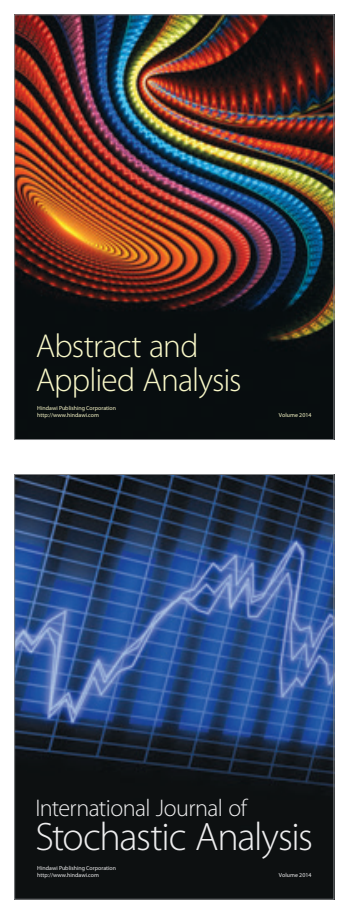

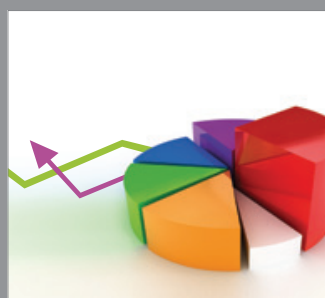

ournal of

Probability and Statistics

Promensencen
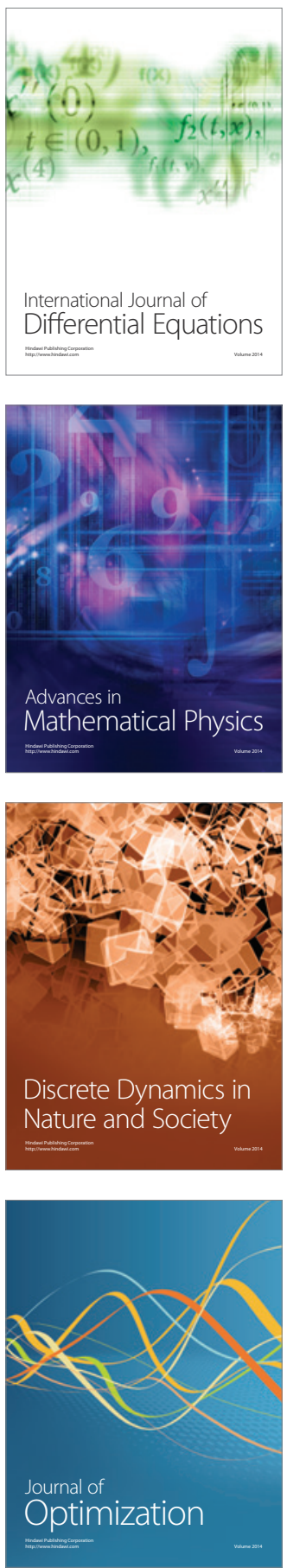